\title{
Fucoidan-Vitamin C complex suppresses tumor invasion through the basement membrane, with scarce injuries to normal or tumor cells, via decreases in oxidative stress and matrix metalloproteinases
}

\author{
YASUKAZU SAITOH, YUKO NAGAI and NOBUHIKO MIWA \\ Cell-Death Control BioTechnology Laboratory, Faculty of Life and Environmental Sciences, \\ Prefectural University of Hiroshima, Nanatsuka 562, Shobara, Hiroshima 727-0023, Japan
}

Received May 15, 2009; Accepted July 9, 2009

DOI: 10.3892/ijo_00000435

\begin{abstract}
Fucoidan (Fucdn) and vitamin C (VC) were saturatedly dissolved in water and lyophilized and thoroughly ethanol-rinsed until no detection for supernatant vitamin $\mathrm{C}$ to form the Fucdn-VC (1:0.23 wt/wt) inclusion body (FucdnVC-IB). Fucdn-VC-IB increased not only VC-stabilizing at $37^{\circ} \mathrm{C}$, but also hydroxyl-radical scavenging as shown by ESR/ spin-trap method, more markedly than a mere mixture of Fucdn:VC (1:0.23 wt/wt). Invasion of human fibrosarcoma cells HT-1080 through the basement membrane was repressed by Fucdn-VC-IB of non-cytotoxic concentrations without significant inhibition to human skin dermal fibroblasts DUMS-16 cells. Fucdn-VC-IB suppressed the invasiveness-related gelatinases MMP-2/9, and diminished reactive oxygen species inside the cytoplasm around the nucleus, in HT-1080 cells as shown by electrophoretic zymography and the redox indicator NBT assay, respectively. Thus Fucdn-VC-IB markedly exhibits antioxidant and MMP-suppressing activities and preferentially inhibited tumor invasion without cytotoxicity to normal cells, and is suggested as a potent tumor-invasion suppressor.
\end{abstract}

\section{Introduction}

It is known that human tumor cells produce reactive oxygen species (ROS) more abundantly than non-transformed cell lines do (1), and elevated oxidative stress has been found in various types of cancer cells (2). A large body of evidence suggests that ROS are related to diverse abilities of cancer

Correspondence to: Dr Nobuhiko Miwa, Cell-Death Control BioTechnology Laboratory, Faculty of Life and Environmental Sciences, Prefectural University of Hiroshima, Nanatsuka, Shobara, Hiroshima 727-0023, Japan

E-mail: miwa-nob@pu-hiroshima.ac.jp

Key words: fucoidan, vitamin $\mathrm{C}$, tumor cell, invasion, reactive oxygen species, matrix metalloproteinase cells which promote cell proliferation, DNA synthesis (3), survival, cellular migration (4), invasion $(5,6)$, tumor metastasis $(7,8)$ and angiogenesis $(9)$. Furthermore, ROS inside cells act as second messengers and play important roles in intracellular signaling cascades which induce and maintain the oncogenic phenotype of cancer cells (1). On the other hand, it is also known that antioxidants can inhibit tumor cell proliferation (10-12), invasion and metastasis (13-15). Thus, these findings suggest the possibility that scavenging of ROS by antioxidant materials can suppress the tumorigenic actions of ROS and the development of certain cancers.

L-Ascorbic acid (vitamin C; VC) is an important antioxidant and an enzyme co-factor (16-18). VC has been suggested to exert anti-tumor action (19-21), inhibit DNA synthesis of cancer cells (10), enhance the tumoricidal action of another anti-tumor agent (22) and inhibit metastatic colony formation (23). Although it is also known that Asc is easily oxidized and lose its antioxidant ability, oxidation-resistant $\mathrm{VC}$ derivatives, such as ascorbic acid (Asc)-2- $O$-phosphate, Asc-6- $O$-palmitate and Asc-2- $O$-phosphate-6- $O$-palmitate have been reported to inhibit tumor invasion and metastasis (13-15).

On the other hand, fucoidan (Fucdn) is a sulfated poly (L-fucopyranose) present in brown marine algae, and has been previously reported to exhibit antioxidative (24), antitumor and anti-metastatic activities (25-28). These findings indicate that combination of $\mathrm{VC}$ and fucoidan is expected to exert anti-cancer activity more marked than that by treatment with $\mathrm{VC}$ or fucoidan alone.

Major causes of mortality such as tumor invasion and metastasis, should be overcome by cancer-recurrence prevention and cancer therapy (29). It is known that the invasion of tumor cells through the basement membranes is a crucial step in the occurrence of metastasis.

Therefore, in the present study, we focused our attention on the tumor invasion process, and evaluated the effects of combination of $\mathrm{VC}$ and fucoidan on the invasiveness of human fibrosarcoma HT-1080 cells. Moreover, we further investigated the effects of fucoidan-vitamin $\mathrm{C}$ inclusion body (FucdnVC-IB), which was produced by critical-point freeze-drying method, against the tumor invasiveness. Our data demonstrated that Fucdn-VC-IB markedly repressed the invasiveness of 
HT-1080 human fibrosarcoma cells through the inhibition of either secretion of MMPs or intracellular ROS generation.

\section{Materials and methods}

Fucoidan-vitamin C inclusion body (Fucdn-VC-IB). Saturated aqueous solution of polysaccharide fucoidan (Fucdn; AH fucoidan 85, Tanglewood Co., Ltd., Hiroshima, Japan) was mixed with saturated aqueous solution of sodium ascorbic acid (VC; Wako Pure Chemical Industries Ltd., Osaka, Japan) at a weight rate of 1:1, vacuum-freeze drying, and gifted by Nippon Medical Souken Co., Ltd. (Yokohama, Japan). Then, non-included VC contained in the lyophilized substance was removed by washing with ethanol until VC became undetectable in the supernatant (for three times). The sediment after repeated ethanol-washing was regarded as Fucdn-VC-IB. The concentration of free- $\mathrm{VC}$ in the supernatant and included VC in Fucdn-VC-IB were detected by an $\alpha, \alpha^{\prime}$-dipyridyl method. The rate of included VC in the Fucdn-VC-IB was $18.7 \%$ (Fucdn: VC = 1:0.23, wt/wt).

Determination of vitamin $C$ (VC; ascorbic acid). Ascorbic acid in water was determined by $\alpha, \alpha^{\prime}$-dipyridyl method as described previously (30). Briefly, sample solutions (500 $\mu \mathrm{l})$ were mixed with a reaction mixture $(500 \mu \mathrm{l})$ containing $17 \%$ of $o-\mathrm{H}_{3} \mathrm{PO}_{4}, 1.6 \%$ of $\alpha, \alpha^{\prime}$-dioyridyl, and $2 \%$ ferric chloride in a disposable plastic tube, and then the reaction mixtures were reacted for $20 \mathrm{~min}$ at $37^{\circ} \mathrm{C}$. The absorbance at $530 \mathrm{~nm}$ was measured with a microplate reader (FLUOstar OPTIMA, BMG Labtech, Offenburg, Germany).

Measurements of hydroxyl radicals by electron spin resonance $(E S R)$. The reactivity of VC, Fucdn, Fucdn + VC, or FucdnVC-IB with hydroxyl radicals that were generated by Fenton's reaction was measured with an ESR spectrometer (type JESER30, JOEL, Tokyo, Japan). Sample solutions (180 $\mu \mathrm{l})$ and $100 \mu \mathrm{M}$ of $\mathrm{FeSO}_{4}(30 \mu \mathrm{l})$ were mixed in a disposable plastic tube, followed by addition to $0.8 \mathrm{M}$ of 5,5-dimethyl-1-pyrroline $\mathrm{N}$-oxide (DMPO; Labotech, Tokyo, Japan) $(60 \mu \mathrm{l})$ and $1 \mathrm{mM}$ of $\mathrm{H}_{2} \mathrm{O}_{2}(30 \mu \mathrm{l})$. The reaction mixtures were sucked into a quartz flat cell LLC04 (Labotech) and set in the ESR apparatus. ESR measurements of hydroxyl radicals were carried out at 2 min after the addition of $\mathrm{H}_{2} \mathrm{O}_{2}$. The X-band spectrometer was equipped with an ESPRIT computer system at $100 \mathrm{kHz}$ magnetic field modulations. Instrument setting was as follows: magnetic field, $336 \pm 5 \mathrm{mT}$; $4 \mathrm{~mW}$ microwave power; sweep time, $1 \mathrm{~min}$; modulation width, $0.32 \mathrm{mT}$; amp, 400; $0.3 \mathrm{sec}$ time constant, and $20 \mathrm{sec}$ scan time. Intensity of ESR signals was corrected based on $\mathrm{MnO}$ used as an internal standard.

Cell culture. Human fibrosarcoma HT-1080 cells (31) were obtained from Japanese Cancer Research Resource Bank (JCRB), and human skin dermal fibroblasts DUMS-16 were kindly supplied by Dr Masayoshi Namba of Niimi college. Cells were cultured in Eagle's minimum essential medium (MEM; Nissui Seiyaku, Tokyo, Japan) supplemented with $10 \%$ heat-inactivated fetal bovine serum (FBS, Biological Industries Ltd., Beit Haemek, Israel), and $2 \mathrm{mM} \mathrm{L-glutamine.}$ Cells were cultured at $37^{\circ} \mathrm{C}$ in a humidified atmosphere of $95 \%$ air and $5 \% \mathrm{CO}_{2}$.
Invasion assay. Invasiveness of HT-1080 cells into the reconstituted basement membrane Matrigel (BD Biosciences, MA, USA) was determined as described previously (14). Briefly, cells were detached with PBS-EDTA and gently pipetted to form single-cell suspension in culture medium. Then, cells $\left(1.0 \times 10^{6}\right)$ were mixed with culture medium containing indicated concentrations of Fucdn, Fucdn + VC, or Fucdn-VC-IB. Cells were incubated with gentle stirring agitation with a rotator $\left(7\right.$ rounds $/ \mathrm{min}$ ) at $37^{\circ} \mathrm{C}$ for $45 \mathrm{~min}$, then cell suspensions were added to the upper compartments of a filter basket chamber (cell culture insert, BD Biosciences). After 1-h incubation at $37^{\circ} \mathrm{C}$, cells that invaded through the Matrigel-precoated membrane filter with $8-\mu \mathrm{m}$ pores (BD Biosciences) were stained by Diff-Quik (Sysmex, Kobe, Japan) and counted under a microscope.

Evaluation of cell viability. Cell viability was evaluated by trypan blue exclusion assay. Cells $\left(1.0 \times 10^{6}\right)$ were mixed with culture medium containing indicated concentrations of Fucdn, Fucdn + VC, or Fucdn-VC-IB, and incubated with gentle stirring agitation with a rotator $(7$ rounds $/ \mathrm{min})$ at $37^{\circ} \mathrm{C}$ for $105 \mathrm{~min}$. Treated cells were resuspended in phosphatebuffered saline (PBS) containing 0.2\% trypan blue for $5 \mathrm{~min}$. All the stained and unstained cells were counted in 4 squares of a hemocytometer and the percentage of dead cell was calculated. Furthermore, cell 1 viability was also assessed based on mitochondrial enzymatic conversion of WST-1 [2-(4iodophenyl)-3-(4-nitrophenyl)-5-(2.4-disulfophenyl)-2Htetrazolium, sodium salt] (Dojindo, Kumamoto, Japan) to yellowish formazan, which is indicative of viable cells. Cells $\left(1.0 \times 10^{6}\right)$ were mixed with culture medium containing indicated concentrations of Fucdn, Fucdn + VC, or FucdnVC-IB, and incubated with gentle stirring agitation with a rotator $\left(7\right.$ rounds $/ \mathrm{min}$ ) at $37^{\circ} \mathrm{C}$ for $105 \mathrm{~min}$. Treated cells were collected and seeded at a density of $4.0 \times 10^{4}$ cells/well in a 24-well plate. After 24-h incubation, cells were rinsed with phenol-red (PR)-free medium and then incubated for $3 \mathrm{~h}$ in PR-free MEM containing WST-1 reagents (5 mM WST-1 and $0.2 \mathrm{mM} 1$-methoxy PMS) at $37^{\circ} \mathrm{C}$. The absorbance at $450 \mathrm{~nm}$ was measured with a microplate reader (FLUOstar OPTIMA). The cell viability is expressed as a percentage versus that of the control cells.

Zymography. Activities of MMPs by HT1080 cells were analyzed by gelatin zymography. HT1080 cells in confluent culture condition were washed and replenished with culture medium containing indicated concentrations of Fucdn $(0.8 \%)$, Fucdn + VC $(0.984 \%)$, VC $(0.184 \%)$, or Fucdn-VC-IB $(0.984 \%)$ for $24 \mathrm{~h}$. The supernatant of culture medium (conditioned medium) was collected and concentrated by ultrafiltration (Amicon Ultra-4, Nihon Millipore K.K., Tokyo, Japan), and the protein concentrations were determined using the DC protein assay kit (Bio-Rad, Hercules, CA, USA). The conditioned medium were mixed with Tris-glycine SDS sample buffer (Invitrogen Corp., CA, USA) without heating or reduction, and applied to $10 \%$ polyacrylamide gels copolymerized with $0.1 \%$ gelatin (Invitrogen). After electrophoresis, gels were washed for $30 \mathrm{~min}$ at the room temperature in zymogram renaturing buffer (Invitrogen), and then incubated in zymogram developing buffer (Invitrogen) for $16 \mathrm{~h}$ at $27^{\circ} \mathrm{C}$. 
Subsequently, gels were fixed and stained with $10 \%$ methanol and $10 \%$ acetic acid containing $0.5 \%$ Coomassie blue R250. The protease activity was visualized as transparent bands on the stained gel.

Measurement of intracellular ROS. Superoxide generation was detected by nitroblue tetrazolium (NBT) assay (32). NBT (yellow water-soluble) was reduced by superoxide anion radical to form formazan-NBT (dark-blue water-insoluble). Cells were seeded at a density of $4.0 \times 10^{4}$ cells/well in the Matrigel-precoated 24-well plate. After 24-h incubation, the medium was exchanged to fresh cell culture media containing indicated concentrations of Fucdn $(0.8 \%)$, Fucdn + VC $(0.984 \%)$, VC $(0.184 \%)$, or Fucdn-VC-IB $(0.984 \%)$. After 105-min incubation, cells were rinsed with PR-free MEM and incubated in PR-free DMEM containing 0.2\% NBT (Wako Pure Chemical Industries Ltd.) for $90 \mathrm{~min}$. Cells were rinsed with cold PBS, stained and photographed. Then, the formazan was dissolved in $2 \mathrm{M} \mathrm{KOH} / \mathrm{DMSO}$ solution for $30 \mathrm{~min}$ at $37^{\circ} \mathrm{C}$, and the absorbance was determined at $680 \mathrm{~nm}$.

Statistical analysis. Data were expressed as mean $\pm \mathrm{SD}$, and statistical comparisons were performed using an unpaired Student's t-test or Dunnet's multiple comparison test. Differences with $\mathrm{P}<0.05$ were considered to be statistically significant.

\section{Results}

Inhibitory effects of Fundn-Vc-IB on stability of vitamin $C$ against auto-oxidation. Although it is suggested that antioxidative activity of a substance is quite important for its anti-cancer effects, VC is known to be a very unstable substance, and VC is so easily oxidized and lose its antioxidant ability. To examine whether Fucdn-VC-IB can prevent auto-oxidation of $\mathrm{VC}$, we evaluated the temporal stabilities of VC in the medium containing VC, Fucdn + VC, or Fucdn-VC-IB (Fig. 1). The concentration of $\mathrm{VC}$ in the medium containing $\mathrm{VC}$ alone was markedly decreased in a time-dependent manner. However, the concentration of $\mathrm{VC}$ in the medium containing Fucdn + VC or Fucdn-VC-IB was retained at a higher level than that of $\mathrm{VC}$ alone at $37^{\circ} \mathrm{C}$ (Fig. 1A). Thus, Fucdn + VC or Fucdn-VC-IB enhanced the stability of $\mathrm{VC}$ against auto-oxidation at $37^{\circ} \mathrm{C}$. The repressive effects of Fucdn-VC-IB on VC degradation were superior to those of Fucdn $+\mathrm{VC}(\mathrm{P}<0.001)$. In contrast, the concentration of $\mathrm{VC}$ was more drastically decreased in a time-dependent manner, and $\mathrm{VC}$ was not detected after 3-h incubation at $60^{\circ} \mathrm{C}$ (Fig. 1B). There were no differences in VC stabilities among three treatments at $60^{\circ} \mathrm{C}$.

Scavenging effects against hydroxyl radicals. To evaluate the potential of Fucdn-VC-IB as a radical scavenger, ESR (electron spin resonance) spin trapping method was used to assess hydroxyl radicals generated by the Fenton reaction [the treatment of $\mathrm{H}_{2} \mathrm{O}_{2}$ with ferrous ions $\left(\mathrm{Fe}_{2}^{+}\right)$]. When each sample was added into a hydroxyl radical-generation system, the signal intensity of DMPO-OH was recorded. In this measurement, a typical four-line ESR signal indicative of a DMPO-OH adduct (1:2:2:1 quartet) was detected when each of

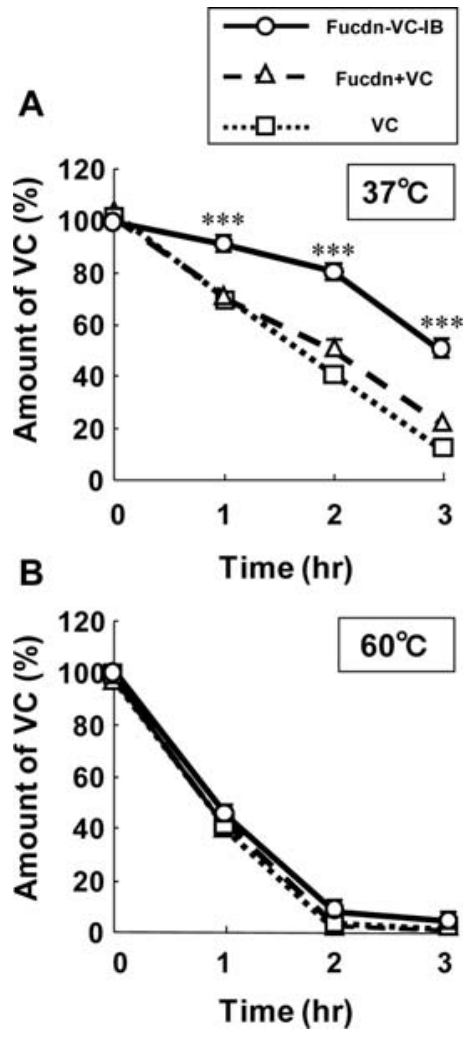

Figure 1. Inhibitory effects of Fucdn-VC-IB formation on auto-oxidation of vitamin C. After preparation of each medium containing VC $(100 \mu \mathrm{M})$, Fucdn $+\mathrm{VC}(100 \mu \mathrm{M})$, or Fucdn-VC-IB $(100 \mu \mathrm{M}$; VC equivalent), they were incubated for $0-3 \mathrm{~h}$ at $37^{\circ} \mathrm{C}(\mathrm{A})$ or $60^{\circ} \mathrm{C}(\mathrm{B})$. Then, the concentration of $\mathrm{VC}$ was determined by $\alpha, \alpha^{\prime}$-dipyridyl method. Significantly different from Fucdn + VC: ${ }^{* * *} \mathrm{P}<0.001$.

the samples was treated with $\mathrm{H}_{2} \mathrm{O}_{2}$ and $\mathrm{Fe}_{2}{ }^{+}$. As shown in Fig. 2, striking scavenging effects against hydroxyl radicals were noted in all the samples (VC, Fucdn, Fucdn + VC, and Fucdn-VC-IB) as compared with Milli-Q water (control). The Fucdn + VC showed higher scavenging effects than that of Fucdn or VC alone. Fucdn-VC-IB showed significant remarkable effects as compared with Fucdn + VC $(\mathrm{P}<0.0001)$. Therefore, these results suggest that Fucdn-VC-IB has a potent scavenging effect against hydroxyl radicals through a mechanism different from simple mixture of Fucdn plus VC.

Inhibitory effects of Fucdn-VC-IB on tumor invasion of human fibrosarcoma HT-1080 cells. It was found that Fucdn, Fucdn + VC, or Fucdn-VC-IB inhibited tumor invasion of human fibrosarcoma HT-1080 cells through the reconstituted basement membrane Matrigel. The number of invasive cells was decreased in all treatments in a dose-dependent manner (Fig. 3). Fucdn-VC-IB was the most effective in inhibition of the effects on tumor invasion out of all the treatments examined, and Fucdn-VC-IB significantly repressed the tumor invasion as compared with Fucdn $+\mathrm{VC}(\mathrm{P}<0.001)$ at doses $<0.984 \%$ (wt/wt).

Effects of Fucdn-VC-IB on cell viability of human fibrosarcoma HT-1080 or human skin dermal fibroblasts DUMS-16 cells. Acute and subacute cytotoxicities of Fucdn, Fucdn + VC, or Fucdn-VC-IB in human fibrosarcoma HT-1080 or human 
A

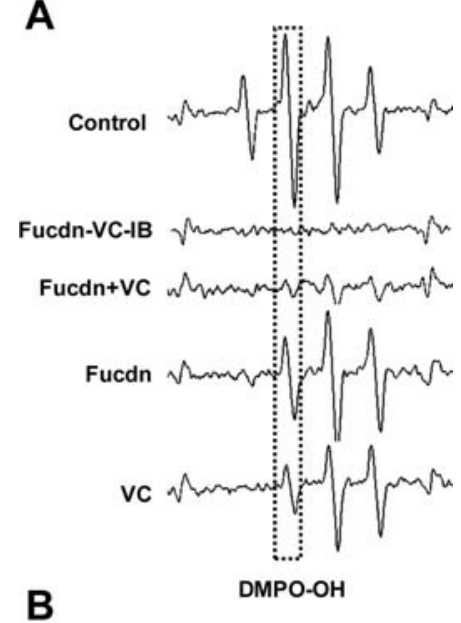

B

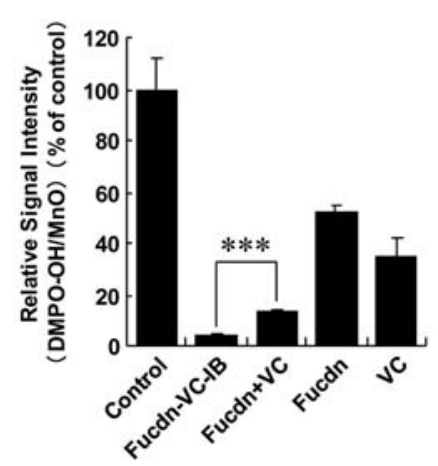

Figure 2. Scavenging effect of Fucdn-VC-IB against hydroxyl radicals. The reactivity of VC $(10 \mu \mathrm{M})$, Fucdn, Fucdn + VC $(10 \mu \mathrm{M})$, or Fucdn-VC-IB (10 $\mu \mathrm{M}$; VC equivalent) with hydroxyl radicals that were generated by Fenton's reaction was measured with an ESR spectrometer. Significantly different from Fucdn + VC: ${ }^{* * *} \mathrm{P}<0.0001$. skin dermal fibroblasts DUMS-16 cells were evaluated by trypan blue exclusion assay (for acute cytotoxicity) and WST-1 assay (for subacute cytotoxicity), respectively. As for estimation of acute cytotoxicity, a significant decrease in cell viability $(\mathrm{P}<0.05)$ was observed only in one dose of Fucdn-VC-IB (1.23\%)-treated DUMS-16 cells (Fig. 4A). As for the subacute cytotoxicity, the significant decreases in cell viability $(\mathrm{P}<0.01)$ were observed not only in $1.23 \%$ of Fucdn + VC-treated and Fucdn-VC-IB-treated DUMS-16 cells, but also $1.23 \%$ of Fucdn-VC-IB-treated HT-1080 cells (Fig. 4B). These results demonstrate that Fucdn-VC-IB did not show cytotoxicity to normal fibroblasts at least at the concentrations $\leq 0.984 \%$. In order to avoid the possibility that Fucdn-VC-IB may reduce tumor invasiveness by influencing the viability of tumor cells, non-cytotoxic dosage of Fucdn-VC-IB (0.984\%) was used in further experiments.

Effects of Fucdn-VC-IB on gelatinase secretion. To investigate whether each MMP-2 and MMP-9 activity was associated with anti-invasive activities of Fucdn-VC-IB, zymogram assay was used to detect enzyme activity in the culture medium harvested after treatment with Fucdn, Fucdn + VC, VC, or Fucdn-VC-IB (Fig. 5). Our results showed that Fucdn-VC-IB suppressed MMP-2 activity to $18 \%$ and MMP-9 activity to $26 \%$ of the control, respectively. Furthermore, these results also demonstrated that Fucdn-VC-IB drastically reduced both MMP-2 and MMP-9 activities relative to other treatments.

Repressive effect of Fucdn-VC-IB on intracellular ROS production in HT-1080 cells. To examine whether Fucdn, Fucdn + VC, VC, or Fucdn-VC-IB can prevent intracellular ROS production in human human fibrosarcoma HT-1080
A

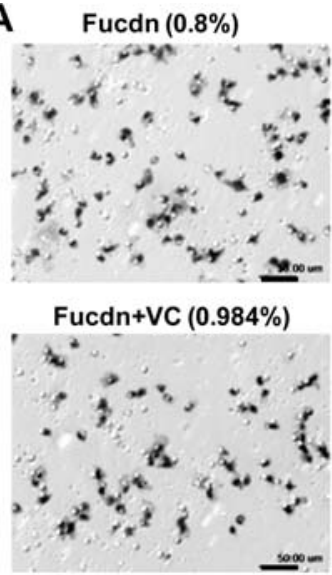

Fucdn-VC-IB (0.984\%)

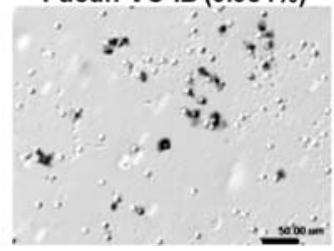

B

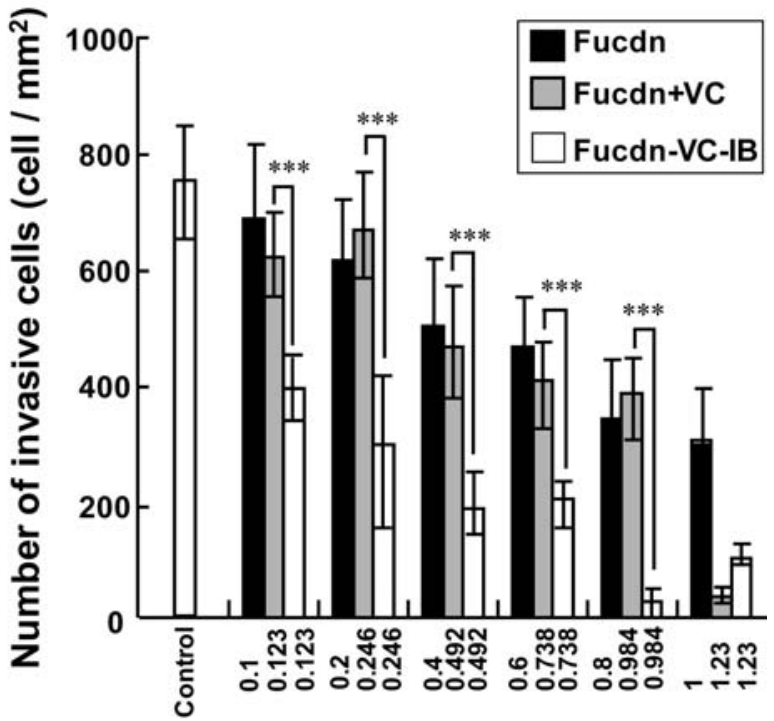

Concentration (\%)

Figure 3. Dose-dependency in inhibitory effects of Fucdn-VC-IB on invasion of human fibrosarcoma HT-1080 cells. Cells were detached to form single-cell suspension in culture medium and treated with indicated concentrations of Fucdn, Fucdn + VC, or Fucdn-VC-IB for 1 h. Then, cell suspensions were added to the upper compartments of a filer basket chamber. After 45 -min incubation at $37^{\circ} \mathrm{C}$, cells that invaded through the Matrigel ${ }^{\mathrm{TM}}$-precoated membrane filter were stained, photographed (A), and counted under a microscope (B). Significantly different from Fucdn $+\mathrm{VC}:{ }^{* *} \mathrm{P}<0.001$. The photographs show stained cells that completed the invasion through the Matrigel-precoated membrane after 1-h incubation. The colorless circles represent 8 - $\mu \mathrm{m}$ diameter pores of the filter that cells had crossed and passed. The scale bar indicates $50 \mu \mathrm{m}$. 
A

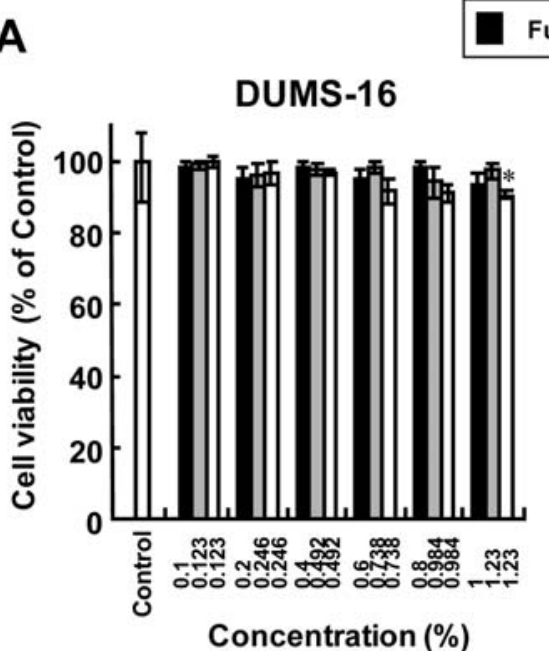

B

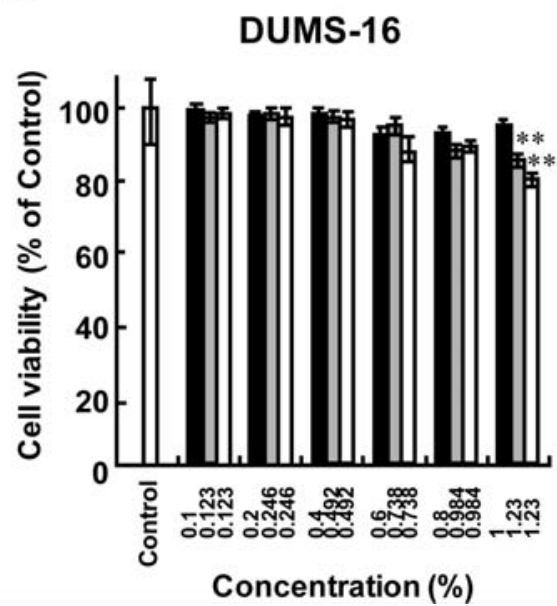

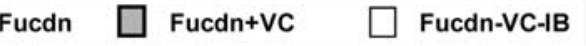

HT-1080

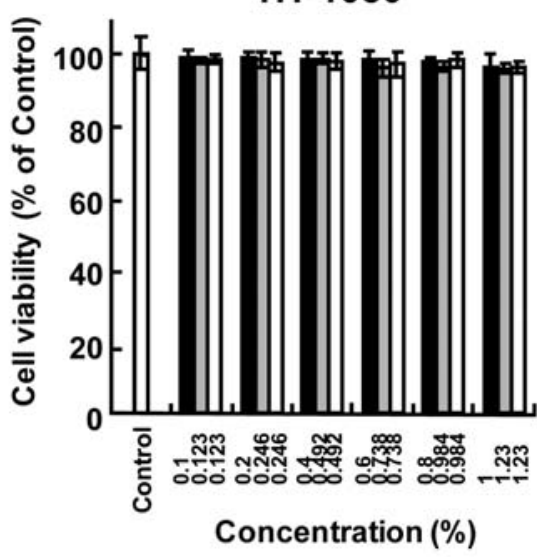

HT-1080

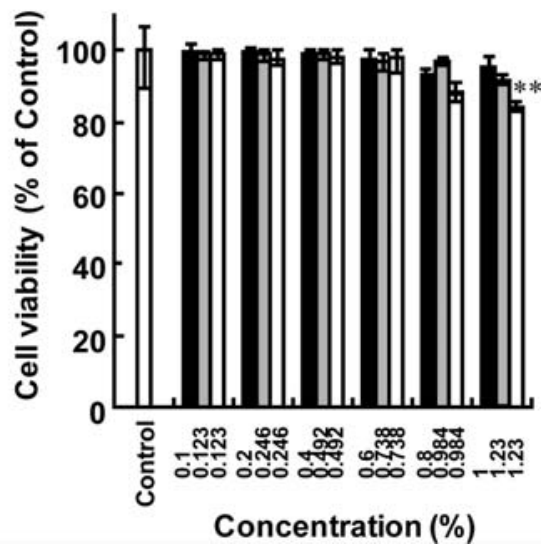

Figure 4. Acute and subacute cytotoxicities of Fucdn-VC-IB on cell viability of human fibrosarcoma HT-1080 or human skin dermal fibroblast DUMS-16 cells. HT-1080 and DUMS-16 cells were detached to form single-cell suspension in culture medium and treated with Fucdn, Fucdn + VC, or Fucdn-VC-IB at the same doses as in Fig. 3, for 105 min when cells contacted each agent in Fig. 3. For estimation of acute cytotoxicity, cell viability was evaluated by trypan blue exclusion assay (A). For estimation of subacute cytotoxicity, the treated cells were seeded on the Matrigel-precoated 24-well plate and grown for 24 h, and cell viability was assessed by WST-1 assay (B). Significantly different from control: ${ }^{*} \mathrm{P}<0.05 ;{ }^{* *} \mathrm{P}<0.01$.

cells, we quantified the intracellular ROS levels by NBT assay. The results showed that intracellular ROS, which was routinely generated in HT-1080 cells, was significantly decreased by Fucdn-VC-IB treatment $(\mathrm{P}<0.001)$, whereas the intracellular ROS levels were not reduced by other treatments (Fig. 6). These results suggest that Fucdn-VC-IB has an excellent repressive effect against intracellular ROS as compared to other treatments.

\section{Discussion}

In the present study, we examined whether combination of $\mathrm{VC}$ and Fucdn exert anti-cancer activity more marked than that from treatment with VC or Fucdn alone, and whether a novel compound named Fucdn-VC-IB achieves an excellent inhibitory effect against invasiveness of HT-1080 human fibrosarcoma cells.

ROS has been suggested to be closely related to diverse abilities of cancer cells such as cell proliferation (3), cellular migration (4), invasion $(5,6)$, metastasis $(7,8)$ and angiogenesis (9). And previous reports suggested that antioxidants exhibited inhibitory effects on tumor cell proliferation (10-12), invasion and metastasis (13-15). Therefore, it is suggested that antioxidative activity of a substance is closely related to its anticancer effects. Specific stability of the antioxidant and the reactivity with ROS are quite important factors to determine the antioxidative activity of the substance. For example, VC derivatives, which are more resistant to auto-oxidation, were reported to show remarkable anti-invasive effects as compared with non-modified VC (14). Although both VC and Fucdn are known to have antioxidative properties $(16,24)$, our results showed that Fucdn-VC-IB was more stable and exhibited more potent antioxidative activity in comparison with other compounds tested (Figs. 1 and 2). These results indicated that Fucdn-VC-IB has a novel property which is different from the simple mixture of VC and Fucdn (Fucdn + VC). Our results clearly demonstrated that FucdnVC-IB, which had a markedly potent antioxidant activity, exhibited the anti-invasive effects more marked than those due to other treatments (Fig. 3).

In addition, Fucdn-VC-IB (at doses $\leq 0.984 \%$ ) did not induce acute or subacute cytotoxicity in either HT-1080 or 

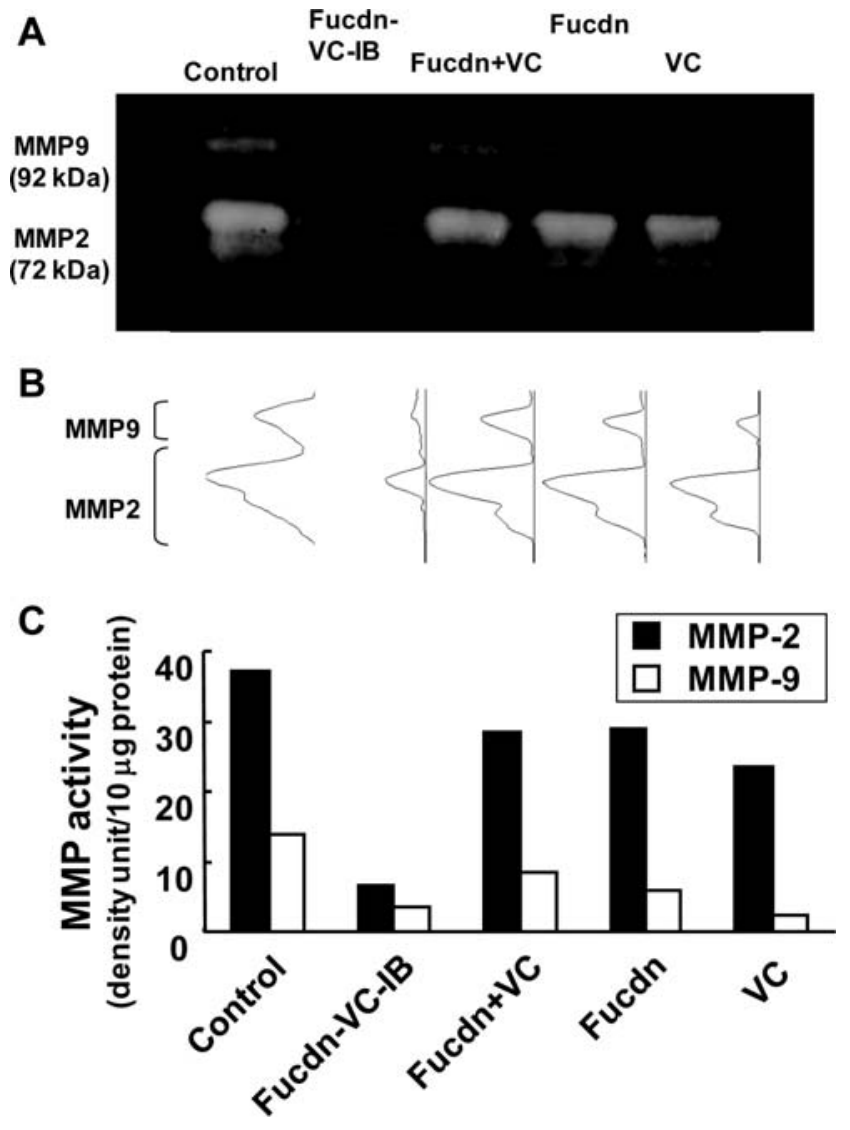

Figure 5. Repressive effects of Fucdn-VC-IB on gelatinase secretion in HT-1080 cells. Cells under a confluent-culture condition were washed and replenished with culture medium containing indicated concentrations of Fucdn $(0.8 \%)$, Fucdn + VC $(0.984 \%)$, VC $(0.184 \%)$, or Fucdn-VC-IB $(0.984 \%)$ for $24 \mathrm{~h}$. The supernatant of culture medium (conditioned medium) was collected and underwent gelatin zymography. The protease activity was visualized as transparent bands on the stained gel (A), and analyzed for densitometry of monochromic electrophorograms (B and C).
DUMS-16 cells (Fig. 4). These results suggest that the repressive effects of Fucdn-VC-IB on tumor invasion are mainly related to suppression of the invasive process, but not the secondary effects through suppression of cell growth or induction of cell deaths. Additionally, since Fucdn-VC-IB (at doses $\leq 0.984 \%$ ) did not induced acute or subacute cytotoxicity in DUMS-16 cells, our results also suggest that Fucdn-VC-IB preferentially act on tumor cells without injuring the normal cells.

The extracellular secretion of proteases plays an important role in migration and invasion of cancer cells (33-36). MMP-2 and MMP-9 are well known as decomposing enzymes for type-IV collagen, which is an important component of the basement membrane. Tumor cells can decompose type-IV collagen and penetrate the basement membrane through secreting of MMP-2 and MMP-9, and the contents of MMP-2 and MMP-9 in tumor cells are related to their invading ability. Hence, we detect whether Fucdn-VC-IB has an influence on the expressions of MMP-2 and MMP-9 in HT-1080 cells. Our result demonstrated that Fucdn-VC-IB markedly decreased MMP-2 and MMP-9 secretions (Fig. 5). Therefore, our results suggested that the repressive effect of Fucdn-VC-IB on tumor invasion is at least partly due to the inhibition of MMPs secretion. Highly invasive or metastatic cancer cells may require a certain level of oxidative stress, and these cells generate large amounts of ROS that are suggested to affect various specific properties of tumor cells such as tumor invasion and metastasis $(8,37)$. Our result indicated that Fucdn-VC-IB significantly reduced the intracellular ROS in HT-1080 cells (Fig. 6). This finding indicates that FucdnVC-IB can suppress intracellular ROS that was routinely generated in HT-1080 cells. Furthermore, previous reports suggested that ROS are implicated in MMP activation and MMP gene expression $(38,39)$. Consequently, our results
A
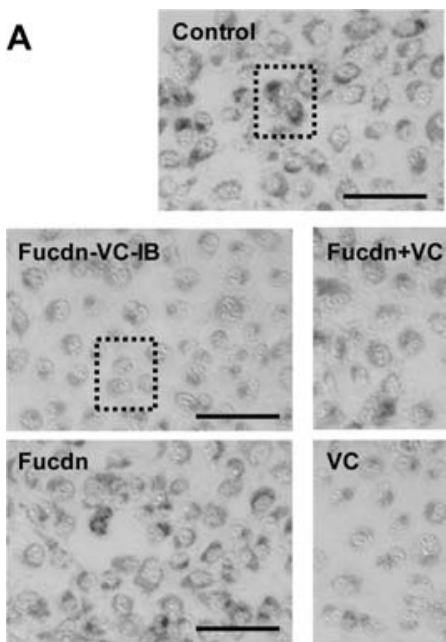

B

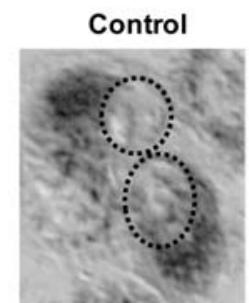

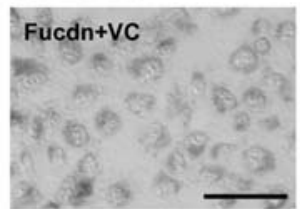

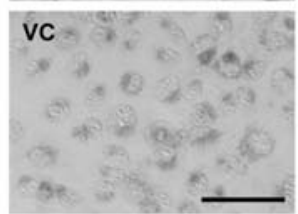

Fucdn-VC-IB

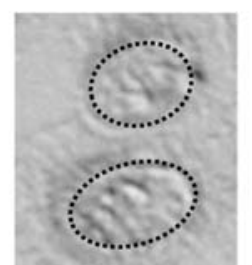

C

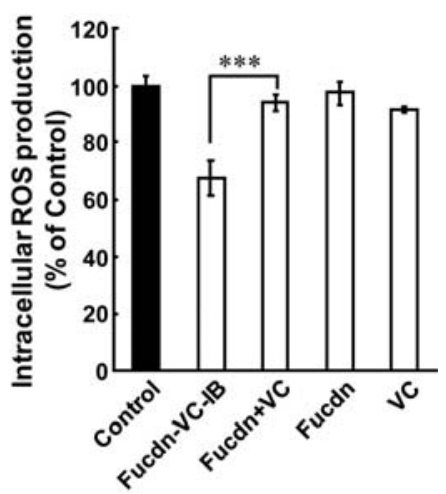

Figure 6. Repressive effects of Fucdn-VC-IB on intracellular superoxide generation in HT-1080 cells. Cells were seeded on a Matrigel-precoated 24well plate, and grown for $24 \mathrm{~h}$. Then, cells were treated with Fucdn $(0.8 \%)$, Fucdn + VC $(0.984 \%)$, VC $(0.184 \%)$, or Fucdn-VC-IB $(0.984 \%)$ for 105 $\mathrm{min}$ as in Fig. 3. Cells were rinsed and incubated for $90 \mathrm{~min}$ in the medium containing $0.2 \%$ NBT. Subsequently, stained cells were photographed under a microscope (A and B), the formazan was extracted, and the absorbance was determined at $680 \mathrm{~nm}(C)$. Significantly different from Fucdn + VC: ${ }^{* * *} \mathrm{P}<0.001$. The scale bar indicates $50 \mu \mathrm{m}$. Areas inside the dashed boxes in (A) were enlarged and shown in (B). Dashed ellipses indicate nuclei. 
suggested that Fucdn-VC-IB inhibited MMP activities through the suppression of intracellular ROS generation, so that FucdnVC-IB achieved the repressive effects on tumor invasion.

In conclusion, the present study demonstrates that FucdnVC-IB exhibits a more prompt antioxidant activity and preferentially inhibits tumor invasion without cytotoxicity to normal cells. Therefore, Fucdn-VC-IB is suggested to be a possible candidate for controlling tumor invasion, and deserves further investigation including in vivo testing.

\section{Acknowledgements}

The authors thank Nippon Medical Souken Co., Ltd. for the gift of the crude preparation containing fucoidan-vitamin C inclusion body, and Ms. Hiroe Masaki and Ms. Saki Fujita for their technical assistance.

\section{References}

1. Szatrowski TP and Nathan CF: Production of large amounts of hydrogen peroxide by human tumor cells. Cancer Res 51 : 794-798, 1991.

2. Behrend L, Henderson G and Zwacka RM: Reactive oxygen species in oncogenic transformation. Biochem Soc Trans 31: 1441-1444, 2003.

3. Amstad P, Crawford D, Muehlematter D, Zbinden I, Larsson R and Cerutti P: Oxidants stress induces the proto-oncogenes, c-fos and c-myc in mouse epidermal cells. Bull Cancer 77: 501-502, 1990.

4. Storz P: Reactive oxygen species in tumor progression. Front Biosci 10: 1881-1896, 2005.

5. Zhang G, Miura Y and Yagasaki K: Suppression of adhesion and invasion of hepatoma cells in culture by tea compounds through antioxidative activity. Cancer Lett 159: 169-173, 2000.

6. Günther S, Ruhe C, Derikito MG, Böse G, Sauer H and Wartenberg M: Polyphenols prevent cell shedding from mouse mammary cancer spheroids and inhibit cancer cell invasion in confrontation cultures derived from embryonic stem cells. Cancer Lett 250: 25-35, 2007.

7. Mukai M, Shinkai K, Tateishi R, Mori Y and Akedo H: Macrophage potentiation of invasive capacity of rat ascites hepatoma cells. Cancer Res 47: 2167-2171, 1987.

8. Nonaka Y, Iwagaki H, Kimura T, Fuchimoto S and Orita K: Effect of reactive oxygen intermediates on the in vitro invasive capacity of tumor cells and liver metastasis in mice. Int J Cancer 54: 983-986, 1993.

9. Maulik $\mathrm{N}$ and Das DK: Redox signaling in vascular angiogenesis. Free Radic Biol Med 33: 1047-1060, 2002.

10. Kageyama K, Onoyama Y, Otani S, Matsui-Yuasa I, Nagao N and Miwa N: Enhanced inhibitory effects of hyperthermia combined with ascorbic acid on DNA synthesis in Ehrlich ascites tumor cells grown at a low cell density. Cancer Biochem Biophys 14: 273-280, 1995

11. Valko M, Leibfritz D, Moncol J, Cronin MT, Mazur M and Telser J: Free radicals and antioxidants in normal physiological functions and human disease. Int J Biochem Cell Biol 9: 44-84, 2007.

12. Saitoh Y, Okayasu H, Xiao L, Harata Y and Miwa N: Neutral $\mathrm{pH}$ hydrogen-enriched electrolyzed water achieves tumorpreferential clonal growth inhibition over normal cells and tumor invasion inhibition concurrently with intracellular oxidant repression. Oncol Res 17: 247-255, 2008.

13. Liu JW, Nagao N, Kageyama K and Miwa N: Antimetastatic and anti-invasive ability of phospho-ascorbyl palmitate through intracellular ascorbate enrichment and the resultant antioxidant action. Oncol Res 11: 479-487, 1999.

14. Liu JW, Nagao N, Kageyama K and Miwa N: Anti-metastatic effect of an autooxidation-resistant and lipophilic ascorbic acid derivative through inhibition of tumor invasion. Anticancer Res 20: $113-118,2000$.

15. Nagao N, Nakayama T, Etoh T, Saiki I and Miwa N: Tumor invasion is inhibited by phosphorylated ascorbate via enrichment of intracellular vitamin $\mathrm{C}$ and decreasing of oxidative stress. J Cancer Res Clin Oncol 126: 511-518, 2000.
16. Levine M: New concepts in the biology and biochemistry of ascorbic acid. N Engl J Med 314: 892-902, 1986.

17. Levine M, Dhariwal KR, Welch RW, Wang Y and Park JB: Determination of optimal vitamin $\mathrm{C}$ requirements in humans. Am J Clin Nutr 62: S1347-S1356, 1995.

18. Sies H and Stahl W: Vitamins E and C, beta-carotene, and other carotenoids as antioxidants. Am J Clin Nutr 62: S1315-S1321, 1995.

19. Bram S, Froussard P, Guichard M, Jasmin C, Augery Y, Sinoussi-Barre F and Wray W: Vitamin C preferential toxicity for malignant melanoma cells. Nature 284: 629-631, 1980.

20. Bissell MJ, Hatie C, Farson DA, Schwarz RI and Soo WJ: Ascorbic acid inhibits replication and infectivity of avian RNA tumor virus. Proc Natl Acad Sci USA 77: 2711-2715, 1980.

21. Leung PY, Miyashita K, Young M and Tsao CS: Cytotoxic effect of ascorbate and its derivatives on cultured malignant and non-malignant cell lines. Anticancer Res 13: 475-480, 1993.

22. Wells WW, Rocque PA, Xu DP, Meyer EB, Charamella LJ and Dimitrov NV: Ascorbic acid and cell survival of adriamycin resistant and sensitive MCF-7 breast tumor cells. Free Radic Biol Med 18: 699-708, 1995.

23. Takenaga M, Igarashi R, Nakayama $T$ and Mizushima $Y$ : Lecithinized ascorbic acid (PC-AS) effectively inhibits murine pulmonary metastasis. Anticancer Res 19: 1085-1091, 1999.

24. Cui YQ, Luo DZ and Wang XM: Fucoidan: advances in the study of its anti-inflammatory and anti-oxidative effects. Yao Xue Xue Bao 43: 1186-1189, 2008.

25. Yamamoto I, Takahashi M, Suzuki T, Seino H and Mori H: Antitumor effect of seaweeds. IV. Enhancement of antitumor activity by sulfation of a crude fucoidan fraction from Sargassum kjellmanianum. Jpn J Exp Med 54: 143-151, 1984.

26. Coombe DR, Parish CR, Ramshaw IA and Snowden JM: Analysis of the inhibition of tumour metastasis by sulphated polysaccharides. Int J Cancer 39: 82-88, 1987.

27. Soeda S, Ishida S, Shimeno H and Nagamatsu A: Inhibitory effect of oversulfated fucoidan on invasion through reconstituted basement membrane by murine Lewis lung carcinoma. Jpn J Cancer Res 85: 1144-1150, 1994.

28. Koyanagi S, Tanigawa N, Nakagawa H, Soeda S and Shimeno H: Oversulfation of fucoidan enhances its anti-angiogenic and antitumor activities. Biochem Pharmacol 65: 173-179, 2003.

29. Shevde LA and Welch DR: Metastasis suppressor pathways - an evolving paradigm. Cancer Lett 198: 1-20, 2003.

30. Zannoni V, Lynch M, Goldstein S and Sato P: A rapid micromethod for the determination of ascorbic acid in plasma and tissues. Biochem Med 11: 41-48, 1974.

31. Rasheed S, Nelson-Rees WA, Toth EM, Arnstein P and Gardner MB: Characterization of a newly derived human sarcoma cell line (HT-1080). Cancer 33: 1027-1033, 1974.

32. Schrenzel J, Serrander L, Banfi B, Nusse O, Fouyouzi R, Lew DP, Demaurex N and Krause KH: Electron currents generated by the human phagocyte NADPH oxidase. Nature 392: 734-737, 1998.

33. Mignatti P and Rifkin DB: Biology and biochemistry of proteinases in tumor invasion. Physiol Rev 73: 161-195, 1993.

34. MacDougall JR and Matrisian LM: Contributions of tumor and stromal matrix metalloproteinases to tumor progression, invasion and metastasis. Cancer Metastasis Rev 14: 351-362, 1995.

35. Johnsen M, Lund LR, R $\notin$ mer J, Almholt K and Dan $\notin$ K: Cancer invasion and tissue remodeling: common themes in proteolytic matrix degradation. Curr Opin Cell Biol 10: 667-671, 1998.

36. Kähäri VM and Saarialho-Kere U: Matrix metalloproteinases and their inhibitors in tumour growth and invasion. Ann Med 31: 34-45, 1999.

37. Loo G: Redox-sensitive mechanisms of phytochemical-mediated inhibition of cancer cell proliferation (review). J Nutr Biochem 14: 64-73, 2003.

38. Rajagopalan S, Meng XP, Ramasamy S, Harrison DG and Galis ZS: Reactive oxygen species produced by macrophagederived foam cells regulate the activity of vascular matrix metalloproteinases in vitro. Implications for atherosclerotic plaque stability. J Clin Invest 98: 2572-2579, 1996.

39. Nelson KK and Melendez JA: Mitochondrial redox control of matrix metalloproteinases. Free Radic Biol Med 37: 768-784, 2004. 